\title{
Experience of microsurgical treatment of brainstem cavernomas: Report of 37 cases
}

\author{
Hao Li, Yan Ju, Bo-wen Cai, Jing Chen, Chao You, Xu-hui Hui
}

Department of Neurosurgery, West China Hospital of Sichuan University, China

\author{
Address for correspondence: \\ Dr. Xu-hui Hui, \\ Department of Neurosurgery, \\ West China Hospital of Sichuan \\ University, 37 Guo Xue Xiang, Wu \\ Hou District, Chengdu 610041, \\ China. \\ E-mail: neurosurgery_Ih@ \\ hotmail.com
}

DOI: $10.4103 / 0028-3886.53269$

\begin{abstract}
Background: Cavernomas are rare in the brainstem and account for $18-35 \%$ of central nervous cavernomas and can cause recurrent hemorrhages, devastating neurological deficits and mortality. Objectives: To summarize the experience of microsurgical treatment of brainstem cavernomas and to investigate curative effect of microsurgical treatment of brainstem cavernomas. Materials and Methods: A retrospective analysis clinical data of 37 patients with brainstem cavernomas seen between 2003 and 2007. The analysis included age distribution, hemorrhage rates, clinical presentation, location of the lesions, and preoperative and postoperative Karnofsky Performance Scale (KPS) scores. The surgical indications, the timing of surgery and the surgical technieues were also assessed. Results: All the 37 patients received microsurgical resections, there was no surgery-related mortality. Histopathological examination confirmed the diagnosis of cavernoma. Postoperatively, 20 patients had functional improvement, 15 patients had no change in the neurological status, and two patients deteriorated. Early surgery was associated with better outcomes. Mean followed up period was 21.5 months (range 6-36 months). During the follow-up 20 patients had resumed activities of daily living (KPS scores of 90-100), 10 patients were able to self-care with some efforts (KPS scores of 70-80), five patients needed considerable assistance (KPS score of 50-60) and two patients suffered hemiparesis (KPS scores of 40). None of the patient had recurrent hemorrhage. Conclusions: Brainstem cavernomas can safely be resected. Successful resection of brainstem cavernomas can be achived by optimal surgical approaches, feasible entry zone and meticulous microsurgical technioues. The goal of surgical intervention should be the total resection of the lesion without any deteriorative in the neurological deficits.
\end{abstract}

Key words: Brainstem, cavernoma, cavernous malformation, microsurgery

\section{Introduction}

Cavernomas, are common in the cerebral hemispheres and rare in the brainstem., ${ }^{[1,2]}$ They account for $5-10 \%$ of all vascular malformations in the central nervous system (CNS). Brainstem cavernomas (BCs) account for $18-35 \%$ of CNS cavernomas ${ }^{[3,4]}$ and can present with hemorrhage or progressive neurological deficit. They can result in death also. ${ }^{[2]}$ In this paper, we summarized our experience of microsurgical treatment of brainstem cavernomas and discussed the most important factors of successful resection of the lesion.

\section{Materials and Methods}

\section{Patients and clinical characteristics}

A retrospective analysis case records of 37 patients with brainstem cavernoma who underwent surgicalintervention in our department between September 2003 and August 2007 was done. There were 25 females and 12 males and from the mean age was 36.5 years (range $18-58$ years). Of the 37 patients, $26(70.3 \%)$ patients had one hemorrhagic episode and the remaining 11 patients had more than one hemorrhagic episode. All patients presented with at least one hemorrhagic episode at the time of admission. The clinical presentations in all patients included hemiparesis, hemianesthesia, ataxia, vertigo, diplopia, cranial nerves V and VIII palsy, dysphagia, symptoms of elevated intracranial pressure (ICP) and intractable hiccup. Preoperatively Karnofsky Performance Scale (KPS) scores ranged from 40 to 100. The clinical presentations differed according to the location of the lesion [Table 1]. All patients were diagnosed with cavernous malformation 
by postoperative histopathologic examination, and the follow-up records were analyzed retrospectively.

\section{Radiological findings}

Magnetic resonance imaging (MRI) was obtained in all the patients. Most of the patients had either magnetic resonance angiography (MRA) or digital subtraction angiography (DSA), which were essentially normal. MRI evaluation revealed 10 cavernomas located in the midbrain [Figure 1], 21 in the pons [Figure 2] and six in the medulla oblongata [Figure 3]. The typical findings were "popcorn" or "mulberry" lesion resulting from previous repeated hemorrhages. In the acute phase of hemorrhage, the non-enhanced T1-weighted images revealed a hyperintense signal, and the classic hemosiderin ring around the lesion on T2-weighted sequences, the characteristic features of cavernomas.

\section{Surgical management}

Most patients underwent surgery within one month of hemorrhage. In 28 patients, surgery was performed during the subacute stage after hemorrhage. In the remaining patients, surgery was performed more than one month after the last episode of hemorrhage. No patients had surgery in the acute phase after hemorrhage. We selected the optimal surgical approach based on the location of the lesions, where the lesions reached the parenchymal surface and where was the safe entry zone into the brainstem. Poppen's suboccipital transtentorial approach was performed with lesions located in the tectum of the midbrain $(n=7)$. Patients with lesions in the lateral surface of the midbrain $(n=3)$ or anterolateral surface of pons $(n=3)$ underwent surgery by subtemporal-occipital approach. Lateral suboccipital retrosigmoid approach was used for the lesions located in the lateral pons $(n=5)$. With regard to the lesions located in the posterior pons $(n=13)$ or medulla oblongata $(n=4)$ which could be best exposed through the floor of the fourth ventricle, thus a suboccipital midline craniotomy was performed and the fourth ventricle was exposed by the transcerebello-medullary approach. Lesions located in the lateral or anterolateral medulla oblongata $(n=2)$ could be exposed satisfactorily by lateral suboccipital transcondylar fossa approach.

The aim of surgery was total resection of the cavernomas while minimizing the damage to the surrounding parenchyma. During the surgical exposure, the hematoma cavity was reached first by a small parenchymal opening and the hematoma was evacuated., Electro-coagulation was used to shrink the lesion followed by limited internal decompression to allow removal of the lesion piecemeal from the cavity of the brainstem. The entire bed of cavity was inspected for residual lesion. Neuronavigation was used for identifying the location of the cavernomas. Electrophysiological stimulation of the resection bed and floor of the fourth ventricle was repeated to confirm preservation of brainstem nuclei.
Table 1: Summary of the neurological deficits of 37 brainstem cavernomas according to the location

\begin{tabular}{lccc}
\hline Signs and symptoms & Midbrain(10) & Pons(21) & $\begin{array}{c}\text { Medulla } \\
\text { oblongata(6) }\end{array}$ \\
\hline Hemiparesis & 1 & 5 & 2 \\
Hemihypesthesia & 2 & 10 & 5 \\
Ataxia & 0 & 7 & 0 \\
Vertigo & 0 & 2 & 0 \\
Diplopia & 2 & 3 & 0 \\
Cranial nerve palsy V VIII & 0 & 16 & 1 \\
Dysphagia & 0 & 3 & 1 \\
ICP & 8 & 1 & 0 \\
Intractable hiccup & 0 & 1 & 1 \\
\hline
\end{tabular}

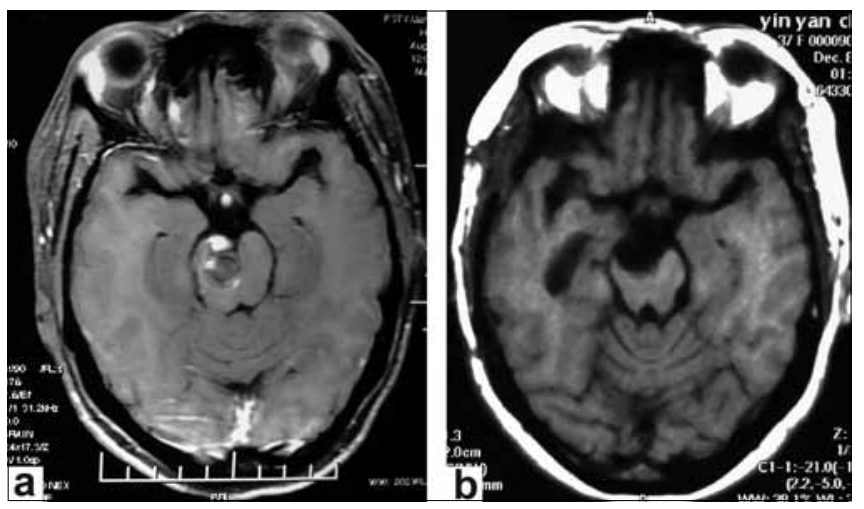

Figure 1: Preoperative axial (a) T1-weighted contrast-enhanced MR image showed a cavernoma in the right anterolateral midbrain region; Postoperative axial (b) T1-weighted MR images showed the cavernoma was completely removed, six months later

\section{Results}

All patients had surgery and total resection of the lesions was achieved in all the patients [Figures 1-3]. There were no surgery-related deaths, neither immediate nor longterm and no patient had postoperative hemorrhage. Histopathological examination of the lesions confirmed the diagnosis of cavernomas. In the immediate postoperative period, eight of the 37 patients, including two patients with midbrain, five patients with pons and one patient with medulla oblongata cavernoma, developed new neurological deficits or had worsening of the preoperative deficits, most of which was transient. Three patients with pons cavernomas and four patients with medulla oblongata cavernoma underwent mechanical ventilation in the immediate postoperative period for a maximu period of $8 \mathrm{~h}$. Postoperatively, two patients (one with pons and one with medulla oblongata cavernoma) received tracheotomy because of IX, X, XI cranial nerve palsy or difficulty in clearing secretions. Five patients (three with pons and two with medulla oblongata cavernoma) needed gastric tube for feeding as they had dysphagia.

Patients who had surgery during the subacute phase after bleed had better outcomes and fewer neurological deficits than those who had surgery more than one 
month after the last episode of bleeding. One month after the surgery, the neurological functional status improved in 20 patients, functional status was similar to the preoperative state in 15 patients and two patients deteriorated. The mean period of follow-up was 21.5 months (range 6-36 months) Tweunty patients recovered completely (KPS scores of 90-100) and resumed activities of daily living, Nine of these patients had normal neurological examination. Ten patients recovered partially (KPS scores of 70-80). Their neurological status was better than preoperative period, and were able to self-care with some efforts. Five patients had the same status as preoperative period (KPS score of 50-60) and needed considerable assistance (KPS score of 50-60)
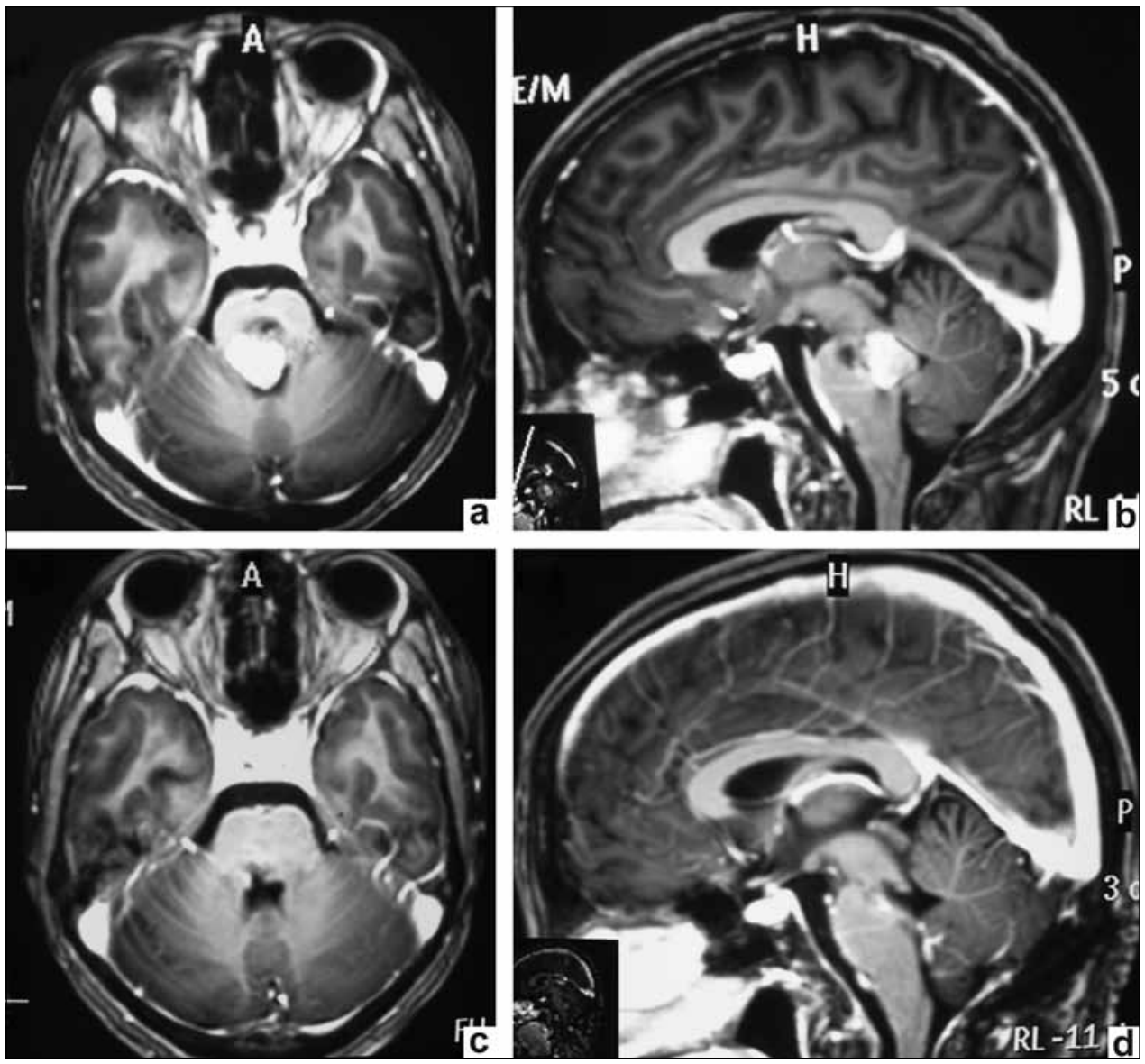

Figure 2: Preoperative axial (a) T1-weighted gadolinium-enhanced and Sagittal (b) MR image demonstrated a cavernoma within the posterior region of the pons, extending into the fourth ventricle; Postoperative axial (c) and Sagittal (d) T1-weighted gadolinium-enhanced MR images demonstrated the complete removal of the lesion, six months later
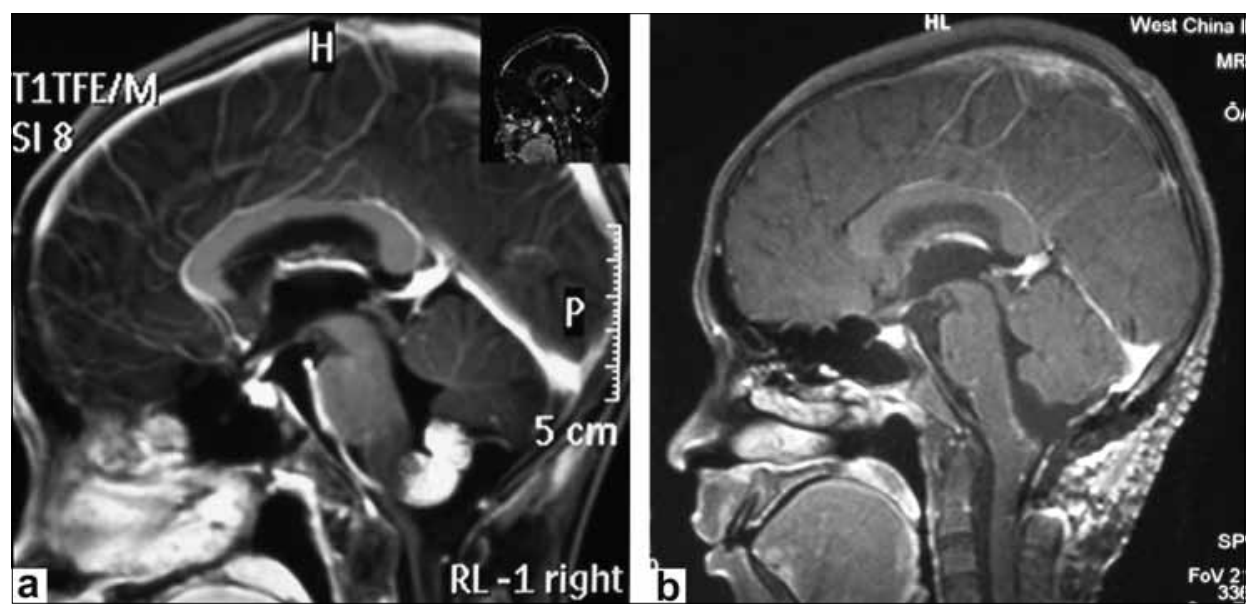

Figure 3: Preoperative sagittal (a) T1-weighted contrast-enhanced MR image showed a cavernoma located in the medulla oblongata region; Postoperative sagittal (b) T1-weighted MR images obtained three months later, demonstrated the cavernoma was completely removed 
and two patients (KPS scores of 40) had hemiparalysis and required special medical care. No patient was in a vegetative state or died and no patient had recurrent hemorrhage.

\section{Discussion}

Cavernomas are uncommon congenital vascular anomalies of the brain characterized by sinusoidal vascular spaces adjacent to each other with no intervening brain parenchyma and occur in any part of the central nervous system (CNS). They account for $5-10 \%$ of all vascular malformations. ${ }^{[5-7]}$ Approximately $10-30 \%$ of intracranial cavernomas are located in the posterior fossa. Brainstem cavernomas are rare and account for fewer than $20 \%$ of intracerebral cavernomas. Approximately $57 \%$ of the cavernomas occur in pons followed by midbrain $(14 \%)$, pontomedullary junction $(12 \%)$, and medulla (5\%). ${ }^{[7-10]}$ Dandy ${ }^{[11]}$ was the first to successfully operate on brainstem cavernomas in 1928.

In our series all the patients had at least one episode of bleeding. The natural history of brainstem cavernomas is different when compared to cavernnnomas in other locations. ${ }^{[12]}$ The annual rate of bleeding risk is much higher in brainstem cavernomas than in other CNS cavernomas. After the initial bleed, the annual rebleeding rate increases significantly regardless of the location of the cavernoma. ${ }^{[13-15]}$ The risk of hemorrhage in patients with brainstem cavernoma is approximately $0.7 \%$ per patientyear of life, and the rebleeding rate is $30 \%$ per year. ${ }^{[7,12]}$ Patients with brainstem cavernoma frequently present with repeated hemorrhages (more than $79 \%$ ) resulting in severe progressive neurological deficits. ${ }^{[9,11]}$ Rcurrent bleeding episodes increase the possibility of a permanent neurological impairment and death. ${ }^{[16]}$ In our study females had higher bleeding rates and young patients and patients with cavernomas of more than $10 \mathrm{~mm}$ size experienced severe bleeding and recurrent bleedings.

MRI scan is the superior imaging modality to CT, DSA and MRA in detecting and delineating brainstem cavernoma. It also provides an indication of the lesion. ${ }^{[17,18,19]}$ Associated pathology like perilesional edema, thrombus and hemorrhage, can be better visualized by MRI. MRI also helps in deffentiating cavernoma bleed from bleeding due other causes like brainstem neoplasm, arteriovenous malformations, infection, hypertension or coagulopathy. ${ }^{[19,20]}$

Surgical resection of brainstem cavernoma is quite challenge for neurosurgeons because the high incidence of postoperative morbidity and mortality. However the major morbidity seems to be mainly related to preoperative brainstem hemorrhages and less to surgical treatment. ${ }^{[12,21,22]}$ Surgical resection has been shown to be feasible and is associated with good clinical outcome. Mostly surgery is suggested for patients with progressive neurologic deficit and bleed. ${ }^{[23,24]}$ Surgery for brainstem cavernomas may be considered in the following indications: (1) recurrent bleeds with progressive neurologic deficits; (2) significant mass effect and progressive enlargement in the follow-up MRI; (3) lesions close to the ventricular or pial surface $(<2 \mathrm{~mm})$; and $(4)$ recurrent bleeds. Surgical complications could be limited by scrupulous planning, careful surgical dissection and making use of neuronavigation and electrophysiological monitoring. It is possible that improved surgical decisionmaking, optimal surgical approach, skilled surgical techniques, and intensive monitoring can optimize the treatment outcomes in brainstem cavernomas.

We recommend ealy surgery in patients with recent bleed. In our study patients who had surgery during the subacute phase after bleed had better outcomes and fewer neurological deficits than those who had surgery more than one month after the last episode of bleeding.

The hematoma may get well organized with the passage of time. This may make it difficult to explore the margins of the cavity and separate cavernoma tissue from reactive gliosis tissue. ${ }^{[25]}$

The surgical approach and parenchymal entry zone for dissection must be guided by the location of the cavernoma and should be individualized for each patient. The viewing angle towards the brainstem depends on a certain surgical approach ${ }^{[26]}$ In our series, the shortest way to the lesions was favored. The surgical approach was chosen based on the location of the cavernoma and its proximity to the ventricle or pial surface. During the surgical procedure, most cavernomas could be visualized on the pial surface or by the color change detected at the surface of the brainstem, the area of that constituted the optimal entry zone, which was a very helpful guide for surgical dissection. ${ }^{[27]}$ Moreover, protection of brainstem tissue and preservation of brainstem function should be the primary concerns. All the brainstem cavernomas should be completely resected, as partial removal will increase the risk of recurrent bleeds. If a venous drainage is noticed during surgery, it should be protected.

Postoperatively of the 37 patients operated, eight (21.6\%) patients experienced surgery-related neurological deterioration. The incidence of new postoperative neurological deficits was higher with pontine cavernomas than with cavernomas located in the midbrain and medulla oblongata. Samii et al., ${ }^{[22]}$ reported new cranial nerve deficits in 17 of the 36 patients $(47.2 \%)$, new motor deficits in three patients $(8.3 \%)$, and new sensory disturbances in 12 patients (33.3\%). Sola et al., ${ }^{[28]}$ reported an incidence of $25 \%$ of new postoperative neurological 
deficits. The postoperative new neurological deficits or worsening of deficits are often transient, and may be caused by changes in microcirculation or edema in the surrounding structures. In our series, the clinical symptoms either improved or remained stable in 35 patients $(94.6 \%)$ postoperatively. The outcome of surgically treated patients was generally good at the time of the last follow-up. In a larger series surgical results were unchanged or improved in $69-91 \% .{ }^{[28-31]}$

Brainstem cavernoms can cause recurrent bleeds, progressive neurological deficits and mortality. Surgical resection should be the favored choice of treatment when the patient suffers bleeding. Surgical resection can be performed safely. Successful resection is based on early surgical decision-making, optimal surgical approach, feasible entry zone, meticulous microsurgical technique, neuronavigation and electrophysiological monitoring. The aim of the surgery is total resection of the lesion while minimizing the damage to the surrounding parenchyma without increasing neurological deficits.

\section{References}

1. Moriarity JL, Clatterbuch RE, Rigamonti D. The natural history of cavernous malformations. Neurosurg Clin N Am 1999;10:411-7.

2. Cristini A, Fischer C, Sindou M. Tectal plate cavernoma: A special entity of brainstem cavernomas: Case report. Surg Neurol 2004;61:474-8; discussion 487.

3. Moriarity JL, Wetzel M, Clatterbuck RE, Javedan S, Sheppard JM, Hoenig-Rigamonti K, et al. The natural history of cavernous malformations: A prospective study of 68 patients. Neurosurgery 1999;44:1166-73.

4. Kupersmith MJ, Kalish H, Epstein F, Yu G, Berenstein A, Woo H, et al. Natural history of brainstem cavernous malformations. Neurosurgery 2001;48:47-53; discussion 53-4.

5. Di Roceo C, Iannelli A, Tamburrini G. Cavernous angiomas of the brain stem in children. Pediatr Neurosurg 1997;27:92-9.

6. Houtteville JP. Brain cavernoma: A dynamic lesion. Surg Neurol 1997;48:610-4.

7. Moriarity JL, Clatterbuck RE, Rigamonti D. The natural history of cavernous malformations. Neurosurg Clin N Am 1999;10:411-7.

8. Robinson JR, Awad IA, Little JR. Natural history of the cavernous angioma. J Neurosurg 1991;75:709-14.

9. Abe H. Clinical presentation of vascular malformations in the brainstem: Comparison of angiographically positive and negativetypes. J Neurol Neurosurg Psychiatry 1989;52:167-75.

10. Requena I, Arias M, Lopez-Ibor L, Pereiro I, Barba A, Alonso A, et al. Cavernomas of the central nervous system: Clinical and neuroimaging manifestations in 47 patients. J Neurol Neurosurg Psychiatry 1991;54:590-4.

11. Dandy WE. Venous abnormalities and angiomas of the brain. Arch Surg 1928;17:715-93.
12. Porter RW, Detwiler PW, Spetzler RF, Lawton MT, Baskin JJ, Derksen PT, et al. Cavernous malformations of the brainstem: Experience with 100 patients. J Neurosurg 1999;90:50-8.

13. Fritschi JA, Reulen HJ, Spetzler RF, Zabramski JM. Cavernous malformations of the brainstem. Acta Neurochir 1994;130:35-46.

14. Kondziolka D, Lunsford LD, Kestle JR. The natural history of cerebral cavernous malformations. J Neurosurg 1995;83:820-4.

15. Aiba T, Tanaka R, Koike T, Kameyama S, Takeda N, Komata T. Natural history of intracranial cavernous malformations. J Neurosurg 1995;83:56-9.

16. Bertalanffy H, Benes L, Miyazawa T. Cerebral cavernous in the adult: Review of the literature and analysis of 72 surgically treated patients. Neurosurg Rev 2002;25:1-53.

17. Bourgouin PM, Tampieri D. Multiple occult vascular malformations of the brain and spinal cord: MRI diagnosis. Neuroradiology 1992;34:110-1.

18. Lynch JC, Andrade R, Pereira C, Salomão JF, Duarte F, Carvalho FG, et al. Angioma cavernoso intracraniano. Arq Neuropsiquiatr $1994 ; 52: 237-42$.

19. Lee RR, Becher MW, Benson ML, Rigamonti D. Brain eapillary telangiectasia: MR imaging appearance and clinicohistopathologic findings. Radiology 1997;205:797-805.

20. Carcao M, Blaser SI, Grant RM, Weksberg R, Siegel-Bartelt J. MRI findings in macrocephaly-cutis marmorata telangiectatica congenita. Am J Med Genet 1998;76:165-7.

21. Ferroli P, Sinisi M, Franzini A, Giombini S, Solero CL, Broggi G. Brainstem cavernomas: Long-term results of microsurgical resection in 52 patients. Neurosurgery 2005;56:1203-12.

22. Samii M, Eghbal A, Carvalo A, Matthies C. Surgical management of brain stem cavernomas. J Neurosurg 2001;95:825-32.

23. Del Curling O, Kelly DL, Elster AD, Craven TE. An analysis of the natural history of cavernous hemangiomas. J Neurosurg 1991;75:702-8.

24. Scott RM, Barnes P, Kupsky W, Adelman LS. Cavernous angiomas of the central nervous system in children. J Neurosurg 1992;76:38-46.

25. Mathiesen T, Edner G, Kihlstrom L. Deep and brainstem cavernomas: A consecutive 8-year series. J Neurosurg 2003;99:31-7.

26. Oiwa Y, Nakai K, Masaki Y, Masuo O, Kuwata T, Moriwaki H, et al. Presigmoid approach for cavernous angioma in the pons-technical note. Neurol Med Chir (Tokyo) 2002;42:91-6; discussion 97-8.

27. Steinberg GK, Chang SD, Gewirtz RJ, Lopez JR. Microsurgical resection of brainstem, thalamic, and basal ganglia angiographically occult vascular malformations. Neurosurgery 2000;46:260-71.

28. Sola RG, Pulido P, Pastor J, Ochoa M, Castedo J. Surgical treatment of symptomatic cavernous malformations of the brainstem. Acta Neurochir (Wien) 2007;149:463-70.

29. Fritschi JA, Reulen HJ, Spetzler RF, Zabramski JM. Cavernous malformations of the brain stem: A review of 139 cases. Acta Neurochir (Wien) 1994;130:35-46.

30. Sindou M, Yada J, Salord F. Functional results after microsurgical resection of brain stem cavernous malformations: Retrospective study of a 12 patient series and review of the recent literature. Acta Neurochir (Wien) 2000;142:843-53

31. Sandalcioglu IE, Wiedemayer H, Secer S, Asgari S, Stolke D. Surgical removal of brain stem cavernous malformations: Surgical indications, technical considerations, and results. J Neurol Neurosurg Psychiatry 2002;72:351-5.

Accepted on 26-04-2009

Source of Support: Nil, Conflict of Interest: None declared. 